(A) Check for updates

Cite this: Org. Biomol. Chem., 2018, 16,4108

\section{Synthesis of $N$-[(dialkylamino)methyl]acrylamides and $N$-[(dialkylamino)methyl]methacrylamides from Schiff base salts: useful building blocks for smart polymers $\uparrow$}

\author{
Abdullah Alzahrani, (D) Styliana I. Mirallai, (D) * Benjamin A. Chalmers, Patrick McArdle \\ and Fawaz Aldabbagh (iD **
}

The traditional thermal Mannich reaction is unsuitable for preparing polymerizable $N$-methylene amino substituted acrylamides and methacrylamides. Herein we provide a facile multi-gram high yield synthesis of these monomeric precursors to stimuli-responsive polymers by the addition of acrylamides and methacrylamides onto in situ generated or freshly isolated methylene Schiff base (iminium) salts. The $\mathrm{X}$-ray crystal structure of the hydrated iminium salt, 1-(hydroxymethyl)azocan-1-ium chloride and monomer $\cdot \mathrm{HCl}$ salt ( $\mathrm{N}$-[(azocan-1-yl)methyl]prop-2-enamide hydrochloride) is described.
Received 5th April 2018 Accepted 9th May 2018

DOI: $10.1039 / \mathrm{c} 8 \mathrm{ob} 00811 \mathrm{f}$ rsc.li/obc
Literature routes have used one-pot Mannich condensation of (meth)acrylamide with formaldehyde to generate the Schiff base followed by secondary amine addition (Scheme 1). ${ }^{3-6}$ The reaction operates thermally (at $\sim 80{ }^{\circ} \mathrm{C}$ ), and is inefficient in forming the Schiff base in situ, with the elevated temperature resulting in premature polymerization of the monomer and intermediates. The reaction has the added difficulty of monomer isolation, which requires vacuum distillation from the aqueous reaction mixture.

The most widely studied temperature-responsive polymers are those with lower critical solution temperature (LCST) close to physiological temperature, such as poly( $N$-isopropylacrylamide) and poly( $N, N$-diethylacrylamide $)$ with LCST of $32-34{ }^{\circ} \mathrm{C}$ in water. ${ }^{7-9}$ The $N$-dialkyl amino (including saturated nitrogen heterocycle) of substituted acrylamides and methacrylamides can be reversibly ionized allowing for a $\mathrm{pH}$-response that alters polymer hydrophobicity. ${ }^{9-15}$ Amphiphilic block copolymers comprising such monomers can self-assemble into a variety of nano-objects for use as stimuli-responsive<smiles>[R]C(=C)C(=O)NCN1CCCCC1</smiles>

(b)

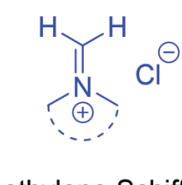
base salt

\title{
$\mathrm{R}=\mathrm{H}$, acrylamide
}

Me, methacrylamide

Fig. 1 (a) Synthetic targets and (b) precursors.

School of Chemistry, National University of Ireland Galway, University Road, Galway, Ireland. E-mail: Styliana.Mirallai@nuigalway.ie

$\dagger$ Electronic supplementary information (ESI) available. CCDC 1819144 and 1819145. For ESI and crystallographic data in CIF or other electronic format see DOI: $10.1039 / \mathrm{c} 80 \mathrm{ob} 00811 \mathrm{f}$

¥Present address: Department of Pharmacy, School of Life Sciences, Pharmacy and Chemistry, Kingston University, Penrhyn Road, Kingston upon Thames, KT1 2EE, UK. E-mail: F.Aldabbagh@kingston.ac.uk.
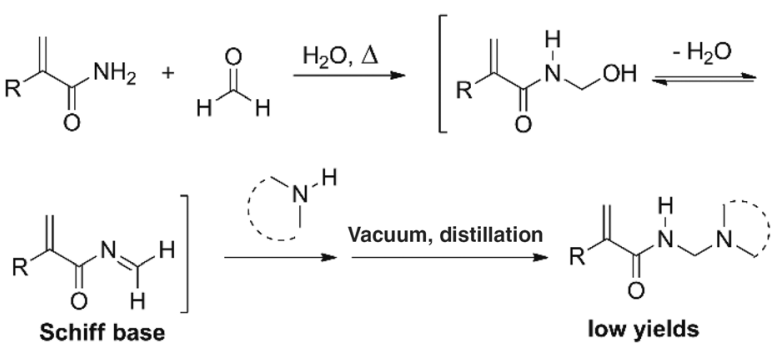

Scheme 1 One-pot thermal Mannich reaction route. 
polymersomes for targeted delivery of therapeutics. ${ }^{11-13}$ In a recent communication, the synthesis of the selected acrylamides containing $\mathrm{N}$-methylene saturated nitrogen heterocycles, and their incorporation into well-defined water-soluble block copolymer polyacrylamides was realised. ${ }^{14}$ In this full paper, we expand on the monomer synthesis by providing efficient multi-gram routes to acrylamides and methacrylamides, including those with dialkyl acyclic and large saturated nitrogen heterocyclic rings. The synthesis involves efficient generation of the methylene Schiff base salt, which was characterized in the hydrated form.

\section{Results and discussion}

In contrast to the one-pot thermal Mannich condensation reaction in Scheme 1, our synthesis uses readily accessible aminals made from the condensation of formaldehyde with secondary amines at $0{ }^{\circ} \mathrm{C}$ (Scheme 2). ${ }^{16,17}$ Böhme pioneered the quaternization of the aminal to generate the Schiff base (iminium) salt, and this procedure using acetyl chloride was followed. ${ }^{17,18}$ There are numerous accounts of alkylation and nucleophilic addition onto methylene Schiff base salts, ${ }^{16-28}$ including by non-vinylic amides. ${ }^{27,28}$ The most utilised is commercial $N, N$ dimethylmethyleneiminium iodide or Eschenmoser's salt. ${ }^{20,29}$ Inspired by the simplicity and low temperatures, acrylamides and methacrylamides were added onto in situ generated methylene Schiff salts to give the monomer hydrochloride salts of morpholine, pyrrolidine and piperidine.

The monomer hydrochloride salts $\mathbf{1 a} \cdot \mathbf{H C l}-\mathbf{3 b} \cdot \mathbf{H C l}$ were precipitated upon the addition of diethyl ether to the reaction in acetonitrile, which allowed the separation of the soluble $N$-acetyl cycloamines (Scheme 2). The isolable $\mathbf{1 a} \cdot \mathbf{H C l}-\mathbf{3 b} \cdot \mathbf{H C l}$ salts are themselves useful as monomeric building blocks in

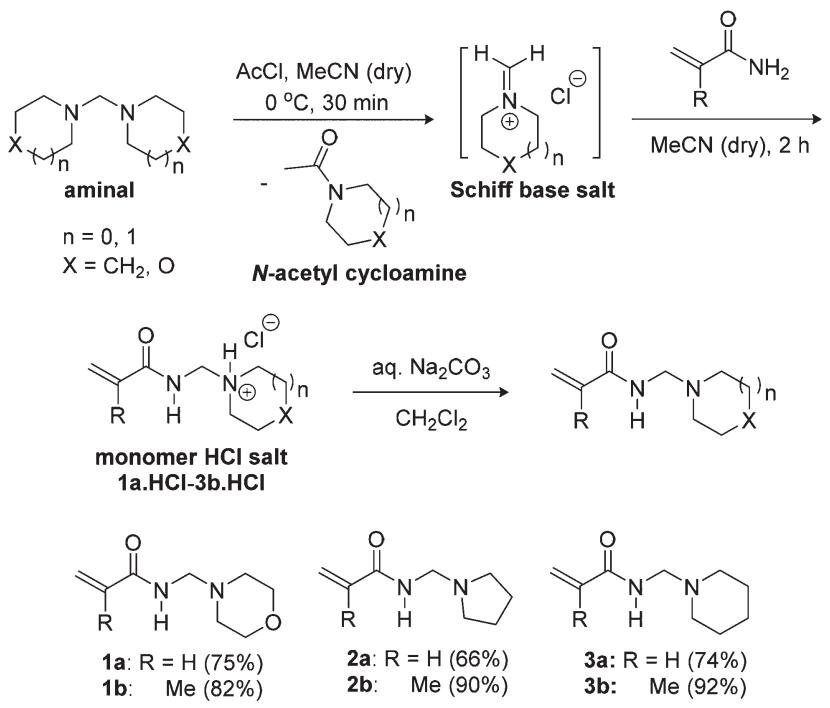

Scheme 2 Synthesis of $N$-[(cycloalkylamino)methyl]acrylamides 1a$3 a,{ }^{14}$ and $N$-[(cycloalkylamino)methyl]methacrylamides $1 b-3 b$ using the in situ Schiff base salt approach. aqueous solution polymerizations. ${ }^{14}$ Basification of the latter allowed the free $N$-[(cycloalkylamino)methyl]acrylamides 1a-3a to be isolated on a $20-25 \mathrm{~g}$ scale in yields of $66-75 \%$ with the $\mathrm{N}$-[(cycloalkylamino)methyl]methacrylamides $\mathbf{1 b}-\mathbf{3 b}$ isolated in higher yields of $82-92 \%$ and on a $\geq 30 \mathrm{~g}$ scale.

Our in situ Schiff base salt approach was not applicable for larger cycloamines (azepane and azocane) and acyclic analogues. Isolation of the methylene Schiff base salts was deemed necessary in these cases due to the poor solubility of their aminals in acetonitrile. Seven and eight-membered heterocyclic base-containing monomers are useful for increased hydrophobicity in the ionisable block segment of amphiphilic copolymers promoting sharper $\mathrm{pH}$-sensitivity of micelles. ${ }^{11,12}$ In contrast, linear dialkyl amine-containing polyacrylamides generally have greater water solubility in comparison to heterocyclic amine-containing analogues affording higher LCSTs. ${ }^{10}$ Treatment of the aminals with acetyl chloride in diethyl ether at $0{ }^{\circ} \mathrm{C}$ allowed access to both larger heterocyclic and acyclic methylene Schiff base salts $\mathbf{4 a - 4 f}$, which were more conveniently characterised as $N$-hydroxymethyl hydrochloride salt derivatives 5a-5f (Scheme 3).

NMR spectra of iminium salts $\mathbf{4 a - 4 f}$ showed mixtures with their respective hydrated derivatives $\mathbf{5 a - 5 f}$. For example, the ${ }^{1} \mathrm{H}$-NMR spectrum in $\mathrm{CD}_{2} \mathrm{Cl}_{2}$ gave similar intensity signals for the exo-methylene of $N$-methylideneazocan-1-ium chloride (4b) at $8.87 \mathrm{ppm}$ and its $N$-hydroxymethyl derivative $\mathbf{5 b}$ exo-methylene at $4.74 \mathrm{ppm}$ (Fig. 2a). Upon recrystallization from acetonitrile, the more stable $N$-(hydroxymethyl)azocan-1-ium chloride 5b was obtained (Fig. 2b). It was thus more convenient to characterize the moisture sensitive methylene Schiff base salts 4a-4f using NMR in $\mathrm{D}_{2} \mathrm{O}$, as 5a-5f (Fig. 2c). An exception was $\mathrm{N}, \mathrm{N}$-dibutylmethaniminium chloride (4f), which appeared less hygroscopic. The NMR spectrum in $\mathrm{CD}_{2} \mathrm{Cl}_{2}$ contained only trace amounts of hydrated derivative 5f (Fig. 3a). The exomethylene at $8.58 \mathrm{ppm}$ in $\mathrm{CD}_{2} \mathrm{Cl}_{2}$ for the methylene Schiff
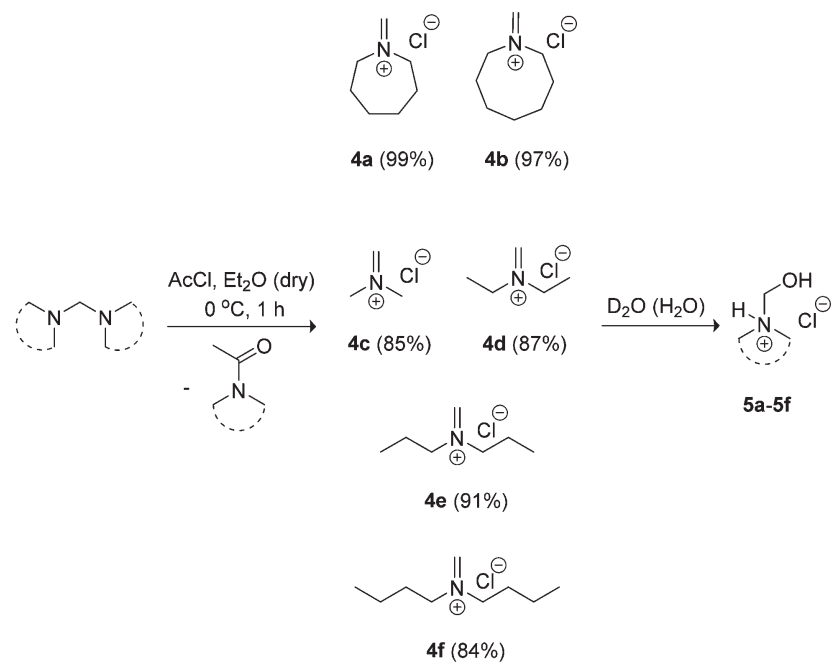

Scheme 3 Synthesis of methylene Schiff base salts $4 a-4 f$ characterised by ${ }^{1} \mathrm{H}$ NMR as hydrated derivatives $5 \mathrm{a}-5 \mathrm{f}$. 


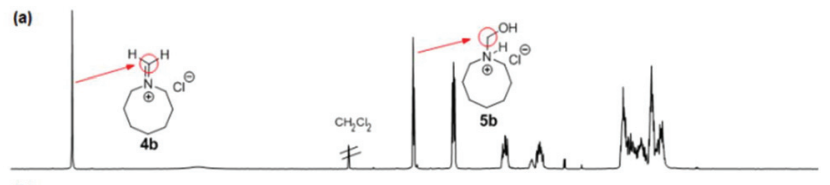

(b) $\mathrm{CH}_{2} \mathrm{Cl}_{2}$

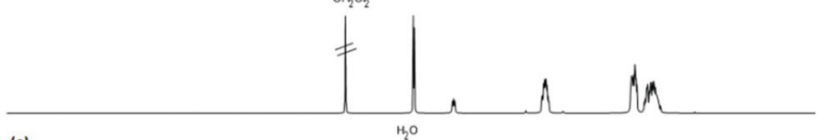

(c)

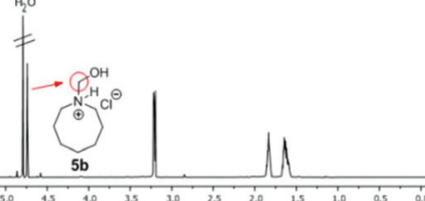

Fig. $2{ }^{1} \mathrm{H}$-NMR spectrum: (a) of the initially isolated mixture containing $\mathrm{N}$-methylideneazocan-1-ium chloride (4b) and $\mathrm{N}$-(hydroxymethyl) azocan-1-ium chloride (5b) in $\mathrm{CD}_{2} \mathrm{Cl}_{2}$ and spectra after recrystallization from $\mathrm{MeCN}$ in (b) $\mathrm{CD}_{2} \mathrm{Cl}_{2}$, and (c) $\mathrm{D}_{2} \mathrm{O}$.

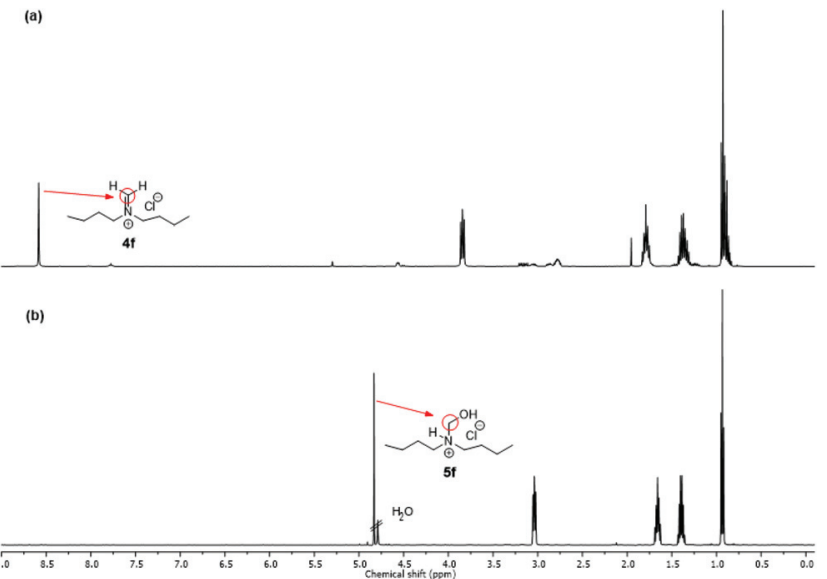

Fig. $3{ }^{1} \mathrm{H}-\mathrm{NMR}$ spectrum: (a) of $\mathrm{N}, \mathrm{N}$-dibutylmethaniminium chloride (4f) in $\mathrm{CD}_{2} \mathrm{Cl}_{2}$ and (b) of $\mathrm{N}$-butyl- $\mathrm{N}$-(hydroxymethyl)butan-1-aminium chloride (5f) in $\mathrm{D}_{2} \mathrm{O}$.

base was replaced by the exo-methylene at $4.83 \mathrm{ppm}$ in $\mathrm{D}_{2} \mathrm{O}$ for $N$-butyl- $N$-(hydroxymethyl)butan-1-aminium chloride (5f in Fig. 3b).

The X-ray crystal structure of $N$-(hydroxymethyl)azocan-1ium chloride (5b) was obtained (Fig. 4 a, Table S1 $\dagger$ ). The large eight membered ring of $\mathbf{5 b}$ was found to be disordered over two equally populated sites with both the $\mathrm{N}-\mathrm{H}$ and $\mathrm{O}-\mathrm{H}$ bonds found to be involved in $\mathrm{H}$-bonding to the chloride counter ion (Fig. 4b). Interestingly, a search of the CCDC database for the $\mathrm{R}_{2} \mathrm{NH}-\mathrm{CH}_{2}-\mathrm{OH}$ moiety gave only one hit, CSD code DIVDET, which was for a pyrimidine salt of tris(hydroxymethyl) ammonium chloride. ${ }^{30}$ The hydroxymethyl hydrochlorides 5a5f were however difficult to isolate cleanly due to their susceptibility to decompose to formaldehyde.

Nucleophilic addition of acrylamides or methacrylamides onto the freshly prepared methylene Schiff base salts of azepane and azocane $\mathbf{4 a}$ and $\mathbf{4 b}$ gave the hydrochloride (a)

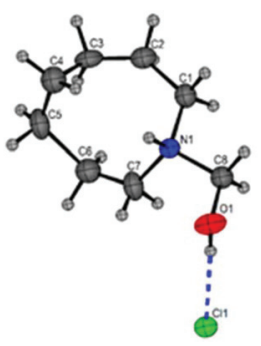

(b)

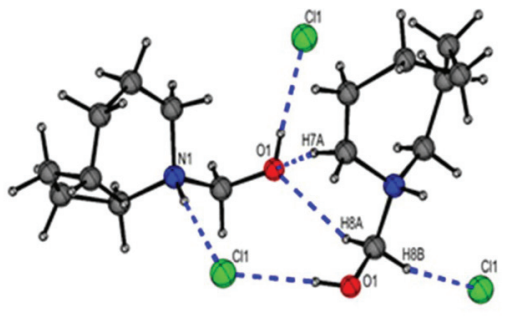

Fig. 4 The X-ray crystal structure of $N$-(hydroxymethyl)azocan-1-ium chloride (5b): (a) only one component of the ring disorder shown for clarity and (b) $\mathrm{H}$-bonding interactions (Table $\mathrm{S} 1 \uparrow$ ).

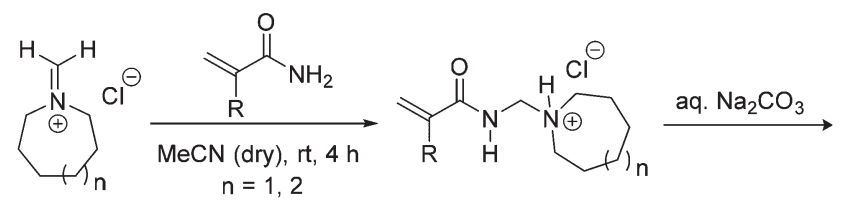

$4 a-4 b$

6a. $\mathrm{HCl}-6 \mathrm{~b} . \mathrm{HCl}$ 7a.HCl-7b. $\mathrm{HCl}$

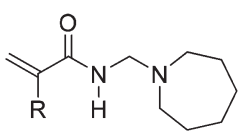

6a: $\mathrm{R}=\mathrm{H}(88 \%)$

6b: $\quad$ Me $(91 \%)$

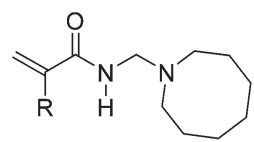

7a: $\mathrm{R}=\mathrm{H}(90 \%)$

7b: $\quad$ Me $(84 \%)$
Scheme 4 Synthesis of seven and eight-membered $N$-[(cycloalkylamino)methyl]acrylamides $6 a-7 a$ and $N$-[(cycloalkylamino)methyl] methacrylamides $6 b-7 b$ from the freshly prepared methylene Schiff base salts.

monomer salts $(\mathbf{6 a} \cdot \mathbf{H C l}, \mathbf{6 b} \cdot \mathbf{H C l}, \mathbf{7 a} \cdot \mathbf{H C l}$ and $\mathbf{7 b} \cdot \mathbf{H C l})$ after precipitation from diethyl ether (Scheme 4). The monomer $\mathrm{HCl}$ salts were suspended in dichloromethane and basified to give the monomers $\mathbf{6 a}-\mathbf{6 b}$ and $7 \mathbf{a}-\mathbf{7 b}$ in high yields of $84-91 \%$ (from $\mathbf{4 a - 4 b}$ ). Attempts to react acrylamide and methacrylamide with an analytically pure sample of $N$-(hydroxymethyl) azocan-1-ium chloride $\mathbf{5 b}$ ) in dried acetonitrile resulted in the isolation of unreacted $\mathbf{5 b}$ and some degradation with the release of formaldehyde. It follows that yields of the monomer from addition onto methylene Schiff base salts were determined by the extent of hydration of the latter substrate.

The X-ray crystal structure of $N$-[(azocan-1-yl)methyl]prop-2enamide hydrochloride $(\mathbf{7} \mathbf{a} \cdot \mathbf{H C l})$ was obtained with very small fitting errors suggesting that the ring was in the optimal conformation (Fig. 5). The crystal structure showed $\mathrm{N}-\mathrm{H}$ bonds forming $\mathrm{H}$-bonding interactions with the chloride anions and the oxygen atoms resulting in intermolecular packing with some weaker $\mathrm{C}-\mathrm{H} \cdots \mathrm{Cl}$ and $\mathrm{C}-\mathrm{H} \cdots \mathrm{O}$ (see Fig. S1, S2 and Table $\mathrm{S} 2 \dagger)$.

For the preparation of $N$-dialkyl amino substituted monomers, $\quad N$-[(dialkylamino)methyl]acrylamides $\mathbf{8 a - 1 1 a}$ and $N$-[(dialkylamino)methyl $]$ methacrylamides $\mathbf{8 b}-\mathbf{1 1} \mathbf{b}$, freshly pre- 


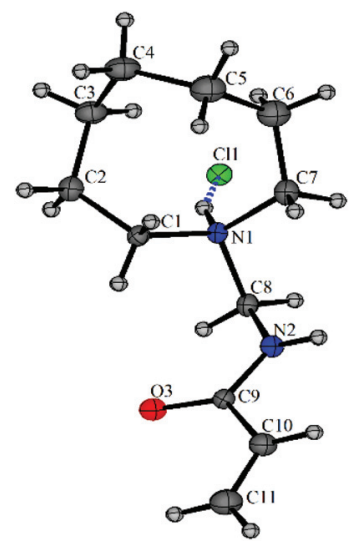

Fig. 5 The X-ray crystal structure of $\mathrm{N}$-[(azocan-1-yl)methyl]prop-2enamide hydrochloride $(7 \mathrm{a} \cdot \mathrm{HCl})$ with one molecule from the asymmetric unit shown.

pared acyclic Schiff base salts $\mathbf{4 c - 4 f}$ were reacted with acrylamides and methacrylamides in acetonitrile at room temperature (Scheme 5). In this case, the isolation of the $\mathrm{N}$-dialkyl amino substituted monomer hydrochloride salts proved difficult due to appreciable solubility in the reaction solvent and attempted precipitation solvents (including diethyl ether). Thus basification of the reaction mixture was preferred and the free monomer bases were isolated in multi-gram quantities (22-49 g) in yields of $69-90 \%$ without the isolation of the intermediate salts.

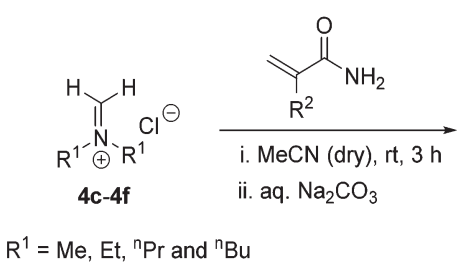

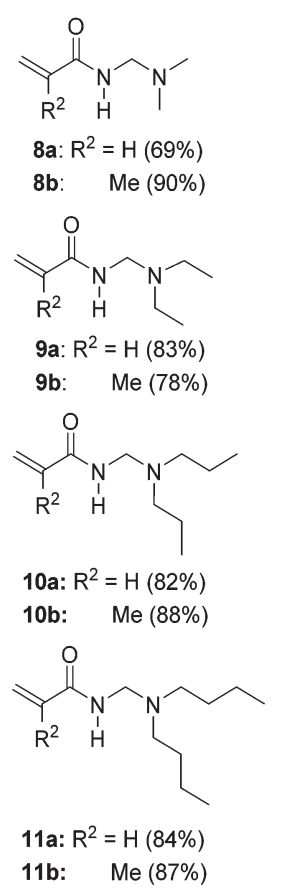

Scheme 5 Synthesis of $N$-[(dialkylamino)methyl]acrylamides $8 a-11 a$ and $N$-[(dialkylamino)methyl]methacrylamides $8 \mathrm{~b}-11 \mathrm{~b}$ from the freshly prepared Schiff base salts.

\section{Conclusions}

Readily accessible methylene Schiff base (iminium) salts have allowed the preparation of eighteen previously inaccessible acrylamides and methacrylamides containing methylene $\mathrm{N}$-amino groups (both heterocyclic and acyclic). Heterocyclic substituted monomer syntheses have the added advantage of allowing the isolation of the monomer hydrochloride salt intermediate useful for polymerizations in water. For the preparation of monomers substituted with azepane, azocane, and acyclic derivatives, the iminium salts should be first isolated, prior to reactions with acrylamides and methacrylamides. Syntheses occur at low or ambient temperatures avoiding premature polymerization of vinyl compounds. Iminium salts are however hygroscopic and X-ray crystal structures of the hydrated eight-membered Schiff base salt, 1-(hydroxymethyl)azocan-1-ium chloride (5b), and the related monomer, $\mathrm{N}$-[(azocan-1-yl)methyl]prop-2-enamide hydrochloride (7a·HCl) are described. Future research will involve controlled radical polymerizations of this new vinyl monomer class to give amphiphilic block copolymers for use as smart stimuli (temperature, $\mathrm{pH}, \mathrm{CO}_{2}$ )-responsive materials.

\section{Experimental}

\section{General information}

Melting points were measured on a Stuart Scientific SMP1 melting point apparatus. Infrared spectra were recorded using a PerkinElmer Spec 1 with ATR attached. ${ }^{1} \mathrm{H}$ NMR spectra were recorded at 400 or $500 \mathrm{MHz}$ and ${ }^{13} \mathrm{C}$ NMR were recorded at 101 or $125 \mathrm{MHz}$ using a $400 \mathrm{MHz}$ JEOL ECX and a $500 \mathrm{MHz}$ Varian instrument respectively. The chemical shifts were recorded in ppm relative to $\mathrm{Me}_{4} \mathrm{Si}$. NMR assignments were supported by DEPT. Deuterated solvents were used for homonuclear lock, and the signals were referenced to the deuterated solvent peaks. 1,4-Dioxane was used as a reference for ${ }^{13} \mathrm{C}$ NMR in $\mathrm{D}_{2} \mathrm{O}$. High resolution mass spectra (HRMS) were recorded using an ESI time-of-flight mass spectrometer (TOFMS) in positive mode. The precision of all accurate mass measurements was better than 5 ppm. All reactions were performed under inert conditions.

\section{Materials}

All chemicals were obtained from commercial sources. Aminals, $1,1^{\prime}$-methylenedipyrrolidine, ${ }^{16} \quad 1,1^{\prime}$-methylenedipiperidine, ${ }^{17}$ $1,1^{\prime}$-methylenebis(azepane), ${ }^{31} \quad N, N, N^{\prime}, N^{\prime}$-tetramethylmethanediamine, ${ }^{16} N, N, N^{\prime}, N^{\prime}$-tetraethylmethanediamine, ${ }^{16}$ and $N, N, N^{\prime}, N^{\prime}$ tetrapropyl-methanediamine ${ }^{31}$ were readily prepared in high yields from the reaction of formaldehyde (Sigma-Aldrich, $37 \mathrm{wt} \%$ in $\mathrm{H}_{2} \mathrm{O}$ ) with the appropriate secondary amine according to the literature procedures. Distilled aminals were stored under vacuum and dry atmospheres in desiccators at room temperature. Heptamethyleneimine (Sigma-Aldrich, 98\%), acetyl chloride (AcCl, Sigma-Aldrich, 98\%), acrylamide (SigmaAldrich, 97\%), and methacrylamide (Sigma-Aldrich, 98\%) were 
used as received. $\mathrm{CH}_{2} \mathrm{Cl}_{2}$ (Sigma-Aldrich, $\left.\geq 99 \%\right), \mathrm{CDCl}_{3}$ (Sigma-Aldrich, 99.8 atom\%), $\mathrm{D}_{2} \mathrm{O}$ (Sigma-Aldrich, 99.9 atom\%), $\mathrm{KOH}$ pellets (Sigma-Aldrich, $\geq 85 \%$ ), $\mathrm{Na}_{2} \mathrm{CO}_{3}$ (SigmaAldrich, $\geq 99 \%$ ), and $\mathrm{MgSO}_{4}$ (Sigma-Aldrich, $\geq 99.99 \%$ ) were used as received. The synthesis of $N$-[(morpholin-4-yl)methyl $]$ prop-2-enamide 1a, 2-methyl- $N$-[(morpholin-4-yl)methyl $]$ prop2-enamide 1b, $N$-[(pyrrolidin-1-yl)methyl $]$ prop-2-enamide $\mathbf{2 a}$ and $N$-(piperidin-1-ylmethyl)prop-2-enamide $\mathbf{3 a}$ is included in our recent communication. ${ }^{14}$ For Schiff base salt and monomer synthesis all solvents were freshly distilled, and the reactions were carried out using anhydrous solvents using an inert nitrogen atmosphere. Acetonitrile (MeCN, Sigma-Aldrich, $\geq 99.9 \%$ ) was freshly distilled over $3 \AA$ molecular sieves and then $\mathrm{CaH}_{2}$ (Sigma-Aldrich, 95\%) and $\mathrm{Et}_{2} \mathrm{O}\left(\mathrm{Et}_{2} \mathrm{O}\right.$, SigmaAldrich, $\geq 99.5 \%$ ) were freshly distilled over Na wire and benzophenone (Sigma-Aldrich, 95\%).

\section{Synthesis of $N$-[(cycloalkylamino)methyl $]$ methacrylamides using the in situ Schiff base salt approach}

AcCl $(14.30 \mathrm{~mL}, 0.20 \mathrm{~mol})$ was added over $30 \mathrm{~min}$ to aminal $(0.20 \mathrm{~mol})$ in $\mathrm{MeCN}(40 \mathrm{~mL})$ at $c a .0{ }^{\circ} \mathrm{C}$. Methacrylamide $(17.02 \mathrm{~g}, 0.20 \mathrm{~mol})$ in $\mathrm{MeCN}(40 \mathrm{~mL})$ was added, and stirred at ca. $20{ }^{\circ} \mathrm{C}$ for $2 \mathrm{~h}$. $\mathrm{Et}_{2} \mathrm{O}(200 \mathrm{~mL})$ was added and the hydrochloride salt of the monomer was precipitated, filtered, and dried under vacuum. The hydrochloride salts $(\mathbf{1} \mathbf{b} \cdot \mathbf{H C l}-\mathbf{3 b} \cdot \mathbf{H C l})$ were recrystallized, dried, and characterized. An aqueous solution of $\mathrm{Na}_{2} \mathrm{CO}_{3}(100 \mathrm{~mL}, 3 \mathrm{M})$ was added to a suspension of the hydrochloride salt in $\mathrm{CH}_{2} \mathrm{Cl}_{2}(100 \mathrm{~mL})$ and stirred for $30 \mathrm{~min}$. The organic layer was separated, and the aqueous layer was washed with $\mathrm{CH}_{2} \mathrm{Cl}_{2}(4 \times 250 \mathrm{~mL})$. The combined organic extracts were dried $\left(\mathrm{MgSO}_{4}\right)$, filtered, and evaporated to dryness to give the monomer, which was recrystallized.

2-Methyl- $N$-[( pyrrolidin-1-yl)methyl $]$ prop-2-enamide hydrochloride (2b·HCl). White solid; mp 131-133 ${ }^{\circ} \mathrm{C}$ (recryst. from MeCN); ${ }^{1} \mathrm{H}$ NMR (400 MHz, DMSO- $\left.d_{6}\right) \delta 1.87-1.94(\mathrm{~m}, 7 \mathrm{H})$, 3.05-3.41 (m, 4H), 4.49 (d, J $6.7 \mathrm{~Hz}, 2 \mathrm{H}), 5.55-5.57(\mathrm{~m}, 1 \mathrm{H})$, 5.92-5.94 (m, 1H), 9.33-9.36 (t, $J 6.7 \mathrm{~Hz}, 1 \mathrm{H}, \mathrm{NH}), 10.61-10.94$ (brs, $1 \mathrm{H}, \mathrm{NH}) ;{ }^{13} \mathrm{C}$ NMR (101 MHz, DMSO- $\left.d_{6}\right) \delta 18.4(\mathrm{Me}), 22.9$, 50.4, 56.4, $122.0\left(\right.$ all $\left.\mathrm{CH}_{2}\right), 138.4(\mathrm{C}), 168.8(\mathrm{C}=\mathrm{O})$.

2-Methyl- $N$-[( pyrrolidin-1-yl)methyl $]$ prop-2-enamide (2b). (30.29 g, 90\%), white solid; mp 56-58 ${ }^{\circ} \mathrm{C}$ (recryst. from MeCN); $v_{\max }\left(\right.$ neat $\left.\mathrm{cm}^{-1}\right) 3215,2962,2819,1655,1619,1521(\mathrm{C}=\mathrm{O})$, 1450, 1362, 1356, 1299, 1208, 1134, 1046; ${ }^{1} \mathrm{H}$ NMR $(400 \mathrm{MHz}$, $\left.\mathrm{CDCl}_{3}\right) \delta 1.75-1.79(\mathrm{~m}, 4 \mathrm{H}), 1.96(\mathrm{~s}, 3 \mathrm{H}), 2.62(\mathrm{t}, J 6.4 \mathrm{~Hz}, 4 \mathrm{H})$, 4.24 (dd, $J 0.7,6.2 \mathrm{~Hz}, 2 \mathrm{H}), 5.33-5.34(\mathrm{~m}, 1 \mathrm{H}), 5.69-5.70(\mathrm{~m}$, 1H), 6.17-6.31 (brs, $1 \mathrm{H}, \mathrm{NH}) ;{ }^{13} \mathrm{C} \mathrm{NMR}\left(101 \mathrm{MHz}, \mathrm{CDCl}_{3}\right.$ ) $\delta 18.8(\mathrm{Me}), 23.7,51.0,58.6,119.8\left(\right.$ all $\left.\mathrm{CH}_{2}\right), 140.1$ (C), 168.7 $(\mathrm{C}=\mathrm{O})$; HRMS (ESI) $m / z[\mathrm{M}+\mathrm{H}]^{+}, \mathrm{C}_{9} \mathrm{H}_{17} \mathrm{~N}_{2} \mathrm{O}$ calcd 169.1341 observed 169.1334.

2-Methyl- $N$-[( piperidin-1-yl)methyl $]$ prop-2-enamide hydrochloride (3b·HCl). White solid; mp 143-145 ${ }^{\circ} \mathrm{C}$ (recryst. from MeCN); ${ }^{1} \mathrm{H}$ NMR (400 MHz, DMSO- $\left.d_{6}\right) \delta 1.34-1.76(\mathrm{~m}, 6 \mathrm{H})$, $1.91(\mathrm{~s}, 3 \mathrm{H}), 2.82-2.85(\mathrm{~m}, 2 \mathrm{H}), 3.26-3.29(\mathrm{~m}, 2 \mathrm{H}), 4.42(\mathrm{~d}$, $J 6.6 \mathrm{~Hz}, 2 \mathrm{H}), 5.56(\mathrm{~s}, 1 \mathrm{H}), 5.92(\mathrm{~s}, 1 \mathrm{H}), 9.13(\mathrm{t}, J 6.6 \mathrm{~Hz}, 1 \mathrm{H}$, $\mathrm{NH}), 10.12-10.32$ (brs, $1 \mathrm{H}, \mathrm{NH}) ;{ }^{13} \mathrm{C}$ NMR (101 MHz, DMSO- $d_{6}$ ) $\delta 18.4(\mathrm{Me}), 21.2,22.1,49.7,59.1,121.9\left(\right.$ all $\mathrm{CH}_{2}$ ), 138.4 (C), $168.7(\mathrm{C}=\mathrm{O})$.

2-Methyl- $N$ - $[($ piperidin-1-yl)methyl $]$ prop-2-enamide

(33.54 g, 92\%), white solid; mp 67-69 ${ }^{\circ} \mathrm{C}$ (recryst. from MeCN); $v_{\max }\left(\right.$ neat, $\left.\mathrm{cm}^{-1}\right)$ 3353, 2934, 2852, $1671(\mathrm{C}=\mathrm{O}), 1655,1615$, 1453, 1453, 1440, 1368, 1333, 1306, 1158, 1110, 1034; ${ }^{1} \mathrm{H}$ NMR $\left(400 \mathrm{MHz}, \mathrm{CDCl}_{3}\right) \delta$ 1.38-1.44 (m, 2H), 1.54-1.59 (m, 4H), 1.95 (s, 3H), 2.45-2.59 (m, 4H), 4.10 (d, J $6.4 \mathrm{~Hz}, 2 \mathrm{H}), 5.34(\mathrm{~s}, 1 \mathrm{H})$, 5.71 (s, 1H), 6.29-6.38 (brs, $1 \mathrm{H}, \mathrm{NH}) ;{ }^{13} \mathrm{C} \mathrm{NMR}(101 \mathrm{MHz}$, $\left.\mathrm{CDCl}_{3}\right) \delta 18.8(\mathrm{Me}), 24.2,25.9,51.6,62.4,119.8\left(\right.$ all $\left.\mathrm{CH}_{2}\right), 140.2$ (C), $168.9(\mathrm{C}=\mathrm{O})$; HRMS (ESI) $m / z[\mathrm{M}+\mathrm{H}]^{+}, \mathrm{C}_{10} \mathrm{H}_{19} \mathrm{~N}_{2} \mathrm{O}$ calcd 183.1497 observed 183.1493.

\section{Synthesis of 1,1'-methylenebis(azocane)}

Heptamethyleneimine (15.0 g, $0.13 \mathrm{~mol})$ was added over $30 \mathrm{~min}$ to formaldehyde (37 wt $\%$ in $\mathrm{H}_{2} \mathrm{O}, 6.2 \mathrm{~mL}, 0.07 \mathrm{~mol}$ ) with stirring at $\mathrm{ca} .0^{\circ} \mathrm{C}$. The solution was stirred overnight at ca. $20{ }^{\circ} \mathrm{C}$, after which $\mathrm{KOH}$ pellets were added to form a saturated solution, and stirring was continued for $30 \mathrm{~min} . \mathrm{H}_{2} \mathrm{O}$ $(40 \mathrm{~mL})$ was added and the mixture was extracted with $\mathrm{Et}_{2} \mathrm{O}$ $(4 \times 40 \mathrm{~mL})$. The organic layers were combined and washed with $\mathrm{H}_{2} \mathrm{O}(3 \times 20 \mathrm{~mL})$, dried $\left(\mathrm{MgSO}_{4}\right)$, and evaporated to dryness. Fractional distillation under reduced pressure gave the title compound as a colorless liquid (14.69 g, 88\%); bp 138-140 ${ }^{\circ} \mathrm{C}$ (0.25 mmHg); $v_{\max }$ (neat, $\mathrm{cm}^{-1}$ ) 2915, 2846, 2779, 1657, 1472, 1448, 1358, 1250, 1158, 1094, 1046, 1017; ${ }^{1} \mathrm{H}$ NMR $\left(400 \mathrm{MHz}, \mathrm{CDCl}_{3}\right) \delta$ 1.51-1.68 (m, 20H), 2.53-2.60 (m, 8H), $3.02(\mathrm{~s}, 2 \mathrm{H}) ;{ }^{13} \mathrm{C} \mathrm{NMR}\left(101 \mathrm{MHz}, \mathrm{CDCl}_{3}\right) \delta 26.1,27.9,28.2$, 52.9, 83.8 (all $\mathrm{CH}_{2}$ ); HRMS (ESI) $m / z[\mathrm{M}+\mathrm{H}]^{+}, \mathrm{C}_{15} \mathrm{H}_{31} \mathrm{~N}_{2}$, calcd 239.2487, observed 239.2312.

\section{Synthesis of $N, N, N^{\prime}, N^{\prime}$-tetrabutylmethanediamine}

Dibutylamine $(68.00 \mathrm{~mL}, 0.40 \mathrm{~mol})$ was added over $30 \mathrm{~min}$ to formaldehyde ( $37 \mathrm{wt} \%$ in $\mathrm{H}_{2} \mathrm{O}, 15.00 \mathrm{~mL}, 0.20 \mathrm{~mol}$ ) with stirring at $c a .0^{\circ} \mathrm{C}$. The solution was stirred overnight at $c a .20^{\circ} \mathrm{C}$. $\mathrm{KOH}$ pellets were added to form a saturated solution, and the drying agent was removed by filtration. Fractional distillation under reduced pressure gave the title compound as a colorless liquid (45.98 g, containing about $10 \%$ of suspected (dibutylamino)methanol impurity by ${ }^{1} \mathrm{H}$ NMR); bp $112-114{ }^{\circ} \mathrm{C}$ (0.25 mmHg); ${ }^{1} \mathrm{H}$ NMR (400 MHz, $\left.\mathrm{CDCl}_{3}\right) \delta 0.89$ (t, J 7.2 Hz, $12 \mathrm{H}), 1.28(\mathrm{~m}, 8 \mathrm{H}), 1.33-1.41(\mathrm{~m}, 8 \mathrm{H}), 2.42(\mathrm{t}, J 7.3 \mathrm{~Hz}, 8 \mathrm{H})$, $2.98(\mathrm{~s}, 2 \mathrm{H}) ;{ }^{13} \mathrm{C}$ NMR (101 MHz, $\left.\mathrm{CDCl}_{3}\right) \delta 14.3(\mathrm{Me}), 20.9$, 29.5, 52.0, $75.6\left(\right.$ all $\mathrm{CH}_{2}$ ). HRMS (ESI) $m / z[\mathrm{M}+\mathrm{H}]^{+}, \mathrm{C}_{17} \mathrm{H}_{39} \mathrm{~N}_{2}$ calcd 271.3113 observed 271.3143. The title compound was used without further purification to prepare $\mathrm{N}, \mathrm{N}$-dibutylmethaniminium chloride (4f).

\section{Synthesis of Schiff base salts}

AcCl $(21.33 \mathrm{~mL}, 0.3 \mathrm{~mol})$ was added over $30 \mathrm{~min}$ to a stirred solution of aminal $(0.3 \mathrm{~mol})$ in $\mathrm{Et}_{2} \mathrm{O}(150 \mathrm{~mL})$ at $c a .0{ }^{\circ} \mathrm{C}$, and the resulting white precipitate was stirred for an additional $30 \mathrm{~min}$. $\mathrm{Et}_{2} \mathrm{O}(200 \mathrm{~mL})$ was added and the precipitate was filtered, and dried under vacuum to give the methylene Schiffbase salt (4a-4f). Iminium salts $\mathbf{4 a - 4 f}$ were immediately used 
in addition reactions with acrylamides and methacrylamides due to their hygroscopic nature.

1-Methylideneazepan-1-ium chloride (4a) (white solid, 43.85 g, 99\%). 1-Methylideneazepan-1-ium chloride (4a) (white solid, $43.85 \mathrm{~g}, 99 \%$ ) was characterized as $\mathrm{N}$-(hydroxymethyl) azepan-1-ium chloride (5a)

${ }^{1} \mathrm{H}$ NMR (400 MHz, D 20 ) $\delta$ 1.63-1.66 (m, 4H), 1.78-1.85 (brs, 4H), 3.17-3.21 (m, 4H), 4.77 (d, J $0.8 \mathrm{~Hz}, 2 \mathrm{H}) ;{ }^{13} \mathrm{C}$ NMR (101 MHz, $\mathrm{D}_{2} \mathrm{O}, 1,4$-dioxane added) $\delta$ 25.2, 26.6, 46.8, 82.4 (all $\mathrm{CH}_{2}$ ).

1-Methylideneazocan-1-ium chloride (4b) (white solid, 47.04 g, 97\%). 1-Methylideneazocan-1-ium chloride (4b) (white solid, $47.04 \mathrm{~g}, 97 \%$ ) was characterized as $\mathrm{N}$-(hydroxymethyl) azocan-1-ium chloride (5b)

${ }^{1} \mathrm{H}$ NMR (500 MHz, $\left.\mathrm{D}_{2} \mathrm{O}\right) \delta 1.57-1.67(\mathrm{~m}, 6 \mathrm{H}), 1.81-1.86(\mathrm{~m}$, $4 \mathrm{H}), 3.21$ (t, $J 5.8 \mathrm{~Hz}, 4 \mathrm{H}), 4.74(\mathrm{~s}, 2 \mathrm{H}) ;{ }^{13} \mathrm{C} \mathrm{NMR}(125 \mathrm{MHz}$, $\mathrm{D}_{2} \mathrm{O}, 1$,4-dioxane added) $\delta 23.9,24.8,25.2,45.8,82.4\left(\right.$ all $\mathrm{CH}_{2}$ ).

$\mathrm{N}, \mathrm{N}$-Dimethylmethaniminium chloride $(4 \mathrm{c})^{32}$ (white solid, $23.85 \mathrm{~g}, \mathbf{8 5} \%)$. $N, N$-Dimethylmethaniminium chloride $(\mathbf{4 c})^{32}$ (white solid, $23.85 \mathrm{~g}, 85 \%$ ) was characterized as hydroxy- $N, N$ dimethylmethanaminium chloride $(5 \mathbf{c})$

${ }^{1} \mathrm{H}$ NMR (400 MHz, D $\left.2 \mathrm{O}\right) \delta 2.79(\mathrm{~s}, 6 \mathrm{H}), 4.56(\mathrm{~s}, 2 \mathrm{H}) ;{ }^{13} \mathrm{C}$ NMR (101 MHz, $\mathrm{D}_{2} \mathrm{O}, 1,4$-dioxane added) $\delta 35.2(\mathrm{Me}), 82.4$ $\left(\mathrm{CH}_{2}\right)$.

$N, N$-Diethylmethaniminium chloride $(4 \mathrm{~d})^{33}$ (white solid, $31.74 \mathrm{~g}, \mathbf{8 7 \%}$ ). $N, N$-Diethylmethaniminium chloride $(\mathbf{4 d})^{33}$ (white solid, $31.74 \mathrm{~g}, 87 \%$ ) was characterized as $N$-ethyl- $N$ (hydroxymethyl)ethanaminium chloride (5d)

${ }^{1} \mathrm{H}$ NMR (400 MHz, $\left.\mathrm{D}_{2} \mathrm{O}\right) \delta 1.23(\mathrm{t}, J 7.3 \mathrm{~Hz}, 6 \mathrm{H}), 3.00-3.07$ $(\mathrm{m}, 4 \mathrm{H}), 4.78(\mathrm{~s}, 2 \mathrm{H}) ;{ }^{13} \mathrm{C}$ NMR (101 MHz, $\mathrm{D}_{2} \mathrm{O}, 1,4$-dioxane added) $\delta 11.2(\mathrm{Me}), 42.9,82.4$ (both $\mathrm{CH}_{2}$ ).

$N, N$-Dipropylmethaniminium chloride $(4 \mathrm{e})^{34,35}$ (white solid, $40.85 \mathrm{~g}, 91 \%) . N, N$-Dipropylmethaniminium chloride $(\mathbf{4 e})^{34,35}$ (white solid, $40.85 \mathrm{~g}, 91 \%$ ) was characterized as $N$-(hydroxymethyl)- $N$-propylpropan-1-aminium chloride (5e)

${ }^{1} \mathrm{H}$ NMR (400 MHz, D $\left.\mathrm{O}\right) \delta 0.92(\mathrm{t}, J 7.6 \mathrm{~Hz}, 6 \mathrm{H}), 1.65$ (sext, $J 7.6 \mathrm{~Hz}, 4 \mathrm{H}), 2.95(\mathrm{t}, J 7.6 \mathrm{~Hz}, 4 \mathrm{H}), 4.78(\mathrm{~s}, 2 \mathrm{H}) ;{ }^{13} \mathrm{C} \mathrm{NMR}$ (101 MHz, $\mathrm{D}_{2} \mathrm{O}, 1,4$-dioxane added) $\delta 10.8(\mathrm{Me}), 19.7,49.7$, $82.4\left(\right.$ all $\left.\mathrm{CH}_{2}\right)$.

$\mathrm{N}, \mathrm{N}$-Dibutylmethaniminium chloride $(4 \mathrm{f})^{35}$ (white solid, $44.78 \mathrm{~g}, \mathbf{8 4 \%})$. $N, N$-Dibutylmethaniminium chloride $(\mathbf{4 f})^{35}$ (white solid, $44.78 \mathrm{~g}, 84 \%$ ) was characterized as $N$-butyl- $N$ (hydroxymethyl)butan-1-aminium chloride (5f)

${ }^{1} \mathrm{H}$ NMR (400 MHz, D $\left.2 \mathrm{O}\right) \delta 0.93(\mathrm{t}, J 6.0 \mathrm{~Hz}, 6 \mathrm{H}), 1.39$ (sext, $J 6.0 \mathrm{~Hz}, 4 \mathrm{H}$ ), 1.66 (quint, $J 6.0 \mathrm{~Hz}, 4 \mathrm{H}), 3.04$ (t, $J 6.0 \mathrm{~Hz}, 4 \mathrm{H}$ ), $4.83(\mathrm{~s}, 2 \mathrm{H}) ;{ }^{13} \mathrm{C} \mathrm{NMR}$ (101 $\mathrm{MHz}, \mathrm{D}_{2} \mathrm{O}, 1,4$-dioxane added) $\delta 13.4(\mathrm{Me}), 19.8,28.2,47.9,82.4\left(\right.$ all $\left.\mathrm{CH}_{2}\right)$.

Synthesis of seven and eight-membered $N$-[(cycloalkylamino)methyl]acrylamides and $N$-[(cycloalkylamino)methyl $]$ methacrylamides

A solution of acrylamide or methacrylamide $(0.03 \mathrm{~mol})$ in MeCN (10 mL) was added to a solution of freshly prepared Schiff base salt $\mathbf{4 a}$ or $\mathbf{4 b}(0.04 \mathrm{~mol})$ in $\mathrm{MeCN}(30 \mathrm{~mL})$ and stirred at $c a .20^{\circ} \mathrm{C}$ for $4 \mathrm{~h} . \mathrm{Et}_{2} \mathrm{O}(200 \mathrm{~mL})$ was added and the hydrochloride salt of the monomer was precipitated, filtered, and dried under vacuum. The hydrochloride salts $(\mathbf{6 a} \cdot \mathbf{H C l}-$ $\mathbf{6 b} \cdot \mathbf{H C l}$ and $\mathbf{7 a} \cdot \mathbf{H C l}-\mathbf{7 b} \cdot \mathbf{H C l})$ were recrystallized, dried, and characterized. An aqueous solution of $\mathrm{Na}_{2} \mathrm{CO}_{3}(15 \mathrm{~mL}, 3 \mathrm{M})$ was added to a suspension of the hydrochloride salt in $\mathrm{CH}_{2} \mathrm{Cl}_{2}$ $(20 \mathrm{~mL})$ and left to stir for an additional $30 \mathrm{~min}$ and extracted with $\mathrm{CH}_{2} \mathrm{Cl}_{2}(4 \times 50 \mathrm{~mL})$. The organic layer was dried $\left(\mathrm{MgSO}_{4}\right)$, filtered and evaporated to dryness to give the corresponding monomers $\mathbf{6 a - 6 b}$ and $7 \mathbf{a}-\mathbf{7} \mathbf{b}$.

$\boldsymbol{N}$-[(Azepan-1-yl)methyl]prop-2-enamide hydrochloride (6a·HCl). White solid; mp $136-138{ }^{\circ} \mathrm{C}$ (recryst. from MeCN); ${ }^{1} \mathrm{H}$ NMR (400 MHz, DMSO- $d_{6}$ ) $\delta$ 1.55-1.62 (m, 4H), 1.79-1.82 (m, $4 \mathrm{H}), 3.03-3.09$ (m, 2H), 3.23-3.32 (m, 2H), 4.49 (d, J $6.8 \mathrm{~Hz}$, 2H), 5.80 (dd, J 1.9, $10.2 \mathrm{~Hz}, 1 \mathrm{H}), 6.26$ (dd, J 1.9, $17.2 \mathrm{~Hz}, 1 \mathrm{H})$, 6.41 (dd, $J$ 10.2, $17.2 \mathrm{~Hz}, 1 \mathrm{H}), 9.53$ (t, $J 6.8 \mathrm{~Hz}, 1 \mathrm{H}, \mathrm{NH}$ ), 10.46-10.63 (brs, $1 \mathrm{H}, \mathrm{NH}) ;{ }^{13} \mathrm{C}$ NMR (101 MHz, DMSO- $d_{6}$ ) $\delta$ 22.9, 26.1, 51.3, 59.2, $128.2\left(\right.$ all $\left.\mathrm{CH}_{2}\right), 130.4(\mathrm{CH}), 166.2$ $(\mathrm{C}=\mathrm{O})$.

$\boldsymbol{N}$-[(Azepan-1-yl)methyl]prop-2-enamide (6a). (4.81 g, 88\%) colourless liquid; bp $134-136{ }^{\circ} \mathrm{C}(0.25 \mathrm{mmHg}) ; v_{\max }$ (neat, $\left.\mathrm{cm}^{-1}\right)$ 3278, 2922, 2852, 2852, $1656(\mathrm{C}=\mathrm{O}), 1623,1539,1452$, 1405, 1365, 1309, 1227, 1133, 1080; ${ }^{1} \mathrm{H}$ NMR (400 MHz, $\mathrm{CDCl}_{3}$ ) $\delta 1.52-1.61(\mathrm{~m}, 8 \mathrm{H}), 2.68(\mathrm{t}, J 5.6 \mathrm{~Hz}, 4 \mathrm{H}), 4.23(\mathrm{~d}, J 6.2 \mathrm{~Hz}$, $2 \mathrm{H}), 5.61$ (dd, J 1.6, $10.2 \mathrm{~Hz}, 1 \mathrm{H}), 6.11$ (dd, J 10.2, $17.0 \mathrm{~Hz}, 1 \mathrm{H}$ ), 6.25 (dd, J 1.6, $17.0 \mathrm{~Hz}, 1 \mathrm{H}$ ), 6.31-6.40 (brs, $1 \mathrm{H}, \mathrm{NH}$ ); ${ }^{13} \mathrm{C} \mathrm{NMR}$ $\left(101 \mathrm{MHz}, \mathrm{CDCl}_{3}\right) \delta$ 26.9, 28.6, 53.1, 62.6, $126.7\left(\right.$ all $\left.\mathrm{CH}_{2}\right), 131.1$ $(\mathrm{CH}), 166.1(\mathrm{C}=\mathrm{O})$; HRMS (ESI) $m / z[\mathrm{M}+\mathrm{H}]^{+}, \mathrm{C}_{10} \mathrm{H}_{19} \mathrm{~N}_{2} \mathrm{O}$ calcd 183.1497 observed 183.1516.

$N$-[(Azepan-1-yl)methyl]-2-methylprop-2-enamide hydrochloride (6b·HCl). White solid; $\mathrm{mp} 88-90{ }^{\circ} \mathrm{C}$ (recryst. from THF); ${ }^{1} \mathrm{H}$ NMR (400 MHz, DMSO- $\left.d_{6}\right) \delta$ 1.53-1.64 (m, 4H), 1.79-1.81 (m, 4H), 1.91 (s, 3H), 3.02-3.15 (m, 2H), 3.23-3.30 (m, 2H), 4.46 (d, J 4.3 Hz, 2H), 5.57 (s, 1H), 5.92 (s, 1H), 9.19 (m, 1H, NH), 10.28-10.44 (brs, $1 \mathrm{H}, \mathrm{NH}) ;{ }^{13} \mathrm{C} \mathrm{NMR}(101 \mathrm{MHz}$, DMSO- $\left.d_{6}\right) \delta 18.4(\mathrm{Me}), 22.8,26.2,51.4,59.7,122.0\left(\right.$ all $\left.\mathrm{CH}_{2}\right)$, 138.4 (C), $168.7(\mathrm{C}=\mathrm{O})$.

\section{$N$-[(Azepan-1-yl)methyl]-2-methylprop-2-enamide} (5.36 g, 91\%) colourless liquid; bp 139-141 ${ }^{\circ} \mathrm{C}(0.25 \mathrm{mmHg})$; $v_{\max }\left(\right.$ neat $\left.\mathrm{cm}^{-1}\right)$ 3328, 2923, 2852, 1655, $1616(\mathrm{C}=\mathrm{O}), 1526$, 1373, 1313, 1201, 1133, 1088, 1020; ${ }^{1} \mathrm{H}$ NMR (400 MHz, $\mathrm{CDCl}_{3}$ ) $\delta 1.56-1.68(\mathrm{~m}, 8 \mathrm{H}), 1.96(\mathrm{~s}, 3 \mathrm{H}), 2.72(\mathrm{t}, J 5.5 \mathrm{~Hz}, 4 \mathrm{H}), 4.24(\mathrm{~d}$, $J 6.1 \mathrm{~Hz}, 2 \mathrm{H}), 5.33(\mathrm{~s}, 1 \mathrm{H}), 5.69(\mathrm{~s}, 1 \mathrm{H}), 6.12-6.22$ (brs, $1 \mathrm{H}$, $\mathrm{NH}) ;{ }^{13} \mathrm{C} \mathrm{NMR}\left(101 \mathrm{MHz}, \mathrm{CDCl}_{3}\right) \delta 18.8(\mathrm{Me}), 27.0,28.6,53.2$, 62.7, 119.6 (all $\mathrm{CH}_{2}$ ), $140.3(\mathrm{C}), 168.8(\mathrm{C}=\mathrm{O})$; HRMS (ESI) $\mathrm{m} / \mathrm{z}$ $[\mathrm{M}+\mathrm{H}]^{+}, \mathrm{C}_{11} \mathrm{H}_{21} \mathrm{~N}_{2} \mathrm{O}$ calcd 197.1654 observed 197.1708.

$\boldsymbol{N}$-[(Azocan-1-yl)methyl]prop-2-enamide hydrochloride (7a.HCl). White solid; mp 87-89 ${ }^{\circ} \mathrm{C}$ (recryst. from EtOAc); ${ }^{1} \mathrm{H}$ NMR (400 MHz, DMSO- $\left.d_{6}\right) \delta 1.43-1.70(\mathrm{~m}, 6 \mathrm{H}), 1.76-1.96(\mathrm{~m}$, $4 \mathrm{H}), 3.10-3.16(\mathrm{~m}, 2 \mathrm{H}), 3.22-3.32(\mathrm{~m}, 2 \mathrm{H}), 4.48-4.68(\mathrm{~m}, 2 \mathrm{H})$, 5.69 (d, J 10.1 Hz, 1H), 6.25 (d, J $17.1 \mathrm{~Hz}, 1 \mathrm{H}), 6.43$ (dd, J 10.1, $17.1 \mathrm{~Hz}, 1 \mathrm{H}), 9.58-9.67(\mathrm{~m}, 1 \mathrm{H}, \mathrm{NH}), 10.25-10.38$ (brs, $1 \mathrm{H}$, $\mathrm{NH}) ;{ }^{13} \mathrm{C}$ NMR (101 MHz, DMSO- $\left.d_{6}\right) \delta 22.1,24.0,25.1,48.8$, 59.2, $128.2\left(\right.$ all $\left.\mathrm{CH}_{2}\right), 130.4(\mathrm{CH}), 166.2(\mathrm{C}=\mathrm{O})$.

$\boldsymbol{N}$-[(Azocan-1-yl)methyl]prop-2-enamide (7a). (5.30 g, 90\%) colourless oil; $v_{\max }\left(\right.$ neat, $\left.\mathrm{cm}^{-1}\right) 3328,2917,2850,1657(\mathrm{C}=\mathrm{O})$, 1624, 1536, 1363, 1232, 1162, 1095, 1060; ${ }^{1} \mathrm{H}$ NMR (400 MHz, $\left.\mathrm{CDCl}_{3}\right) \delta$ 1.50-1.62 (m, 10H), 2.62-2.70 (m, 4H), 4.27 (d, 
$J 6.0 \mathrm{~Hz}, 2 \mathrm{H}$ ), 5.65 (d, J 10.2 Hz, 1H), 5.91-6.02 (brs, 1H), 6.10 (dd, $J 10.2,17.0 \mathrm{~Hz}, 1 \mathrm{H}), 6.28$ (d, $J 17.0 \mathrm{~Hz}, 1 \mathrm{H}) ;{ }^{13} \mathrm{C}$ NMR $\left(101 \mathrm{MHz}, \mathrm{CDCl}_{3}\right) \delta 26.1,27.7,28.0,51.6,62.6,126.7\left(\right.$ all $\left.\mathrm{CH}_{2}\right)$, $131.2(\mathrm{CH}), \quad 166.0(\mathrm{C}=\mathrm{O}) ; \operatorname{HRMS}(\mathrm{ESI}) \mathrm{m} / \mathrm{z}[\mathrm{M}+\mathrm{H}]^{+}$, $\mathrm{C}_{11} \mathrm{H}_{21} \mathrm{~N}_{2} \mathrm{O}$, calcd 197.1656, observed 197.1654.

$N$-[(Azocan-1-yl)methyl]-2-methylprop-2-enamide hydrochloride (7b·HCl). White solid; mp 128-130 ${ }^{\circ} \mathrm{C}$ (recryst. from EtOAc); ${ }^{1} \mathrm{H}$ NMR (400 MHz, DMSO- $\left.d_{6}\right) \delta 1.46-1.75(\mathrm{~m}, 6 \mathrm{H})$, 1.81-1.99 (m, 7H), 3.07-3.18 (m, 2H), 3.23-3.35 (m, 2H), 4.45-4.53 (m, 2H), $5.70(\mathrm{~s}, 1 \mathrm{H}), 5.93(\mathrm{~s}, 1 \mathrm{H}), 9.17-9.27$ (brs, $1 \mathrm{H}$, $\mathrm{NH}), 9.94-10.09$ (brs, $1 \mathrm{H}, \mathrm{NH}) ;{ }^{13} \mathrm{C}$ NMR (101 MHz, DMSO- $d_{6}$ ) $\delta 18.4$ (Me), 22.2, 24.0, 25.0, 48.9, 59.8, $122.0\left(\right.$ all $\left.\mathrm{CH}_{2}\right), 138.4$ (C), $168.6(\mathrm{C}=\mathrm{O})$.

$\mathrm{N}$-[(Azocan-1-yl)methyl]-2-methylprop-2-enamide

(7b). $\left(5.30 \mathrm{~g}, 84 \%\right.$ ) colourless oil; $v_{\max }\left(\right.$ neat, $\left.\mathrm{cm}^{-1}\right) 3324,2918,2850$, 1655, $1618(\mathrm{C}=\mathrm{O}), 1523,1452,1363,1201,1162,1095,1060$; ${ }^{1} \mathrm{H}$ NMR (400 MHz, $\left.\mathrm{CDCl}_{3}\right) \delta 1.49-1.59(\mathrm{~m}, 10 \mathrm{H}), 1.93(\mathrm{~s}, 3 \mathrm{H})$, 2.61-2.66 (m, 4H), 4.21 (t, J 4.3 Hz, 2H), $5.28(\mathrm{~s}, 1 \mathrm{H}), 5.64(\mathrm{~s}$, 1H), 6.12-6.26 (brs, $1 \mathrm{H}, \mathrm{NH}) ;{ }^{13} \mathrm{C} \mathrm{NMR} \mathrm{(101} \mathrm{MHz}, \mathrm{CDCl}_{3}$ ) $\delta 18.8(\mathrm{Me}), 26.0,27.7,28.0,51.5,62.7,119.2\left(\right.$ all $\left.\mathrm{CH}_{2}\right), 140.4$ (C), $168.9(\mathrm{C}=\mathrm{O})$; HRMS (ESI) $m / z[\mathrm{M}+\mathrm{H}]^{+}, \mathrm{C}_{12} \mathrm{H}_{23} \mathrm{~N}_{2} \mathrm{O}$, calcd 211.1838, observed 211.1810.

\section{Synthesis of $N$-[(dialkylamino)methyl $]$ acrylamides and $N$-[(dialkyl-amino)methyl $]$ methacrylamides}

A solution of acrylamide or methacrylamide $(0.25 \mathrm{~mol})$ in MeCN (50 mL) was added to a stirred solution of freshly prepared Schiff base salt $\mathbf{4 c - 4 f}(0.25 \mathrm{~mol})$ in MeCN $(50 \mathrm{~mL})$ and stirred at ca. $20{ }^{\circ} \mathrm{C}$ for $3 \mathrm{~h}$. An aqueous solution of $\mathrm{Na}_{2} \mathrm{CO}_{3}$ (150 mL, $3 \mathrm{M}$ ) was added and the solution was stirred for an additional $30 \mathrm{~min}$ and extracted with $\mathrm{CH}_{2} \mathrm{CL}_{2}(4 \times 250 \mathrm{~mL})$. The organic layer was dried $\left(\mathrm{MgSO}_{4}\right)$, filtered and evaporated to give the corresponding acrylamides 8a-11a and methacrylamides 8b-11b.

$N$-[(Dimethylamino)methyl]prop-2-enamide (8a). (22.11 g, $69 \%$ ) colourless liquid; bp 64-66 ${ }^{\circ} \mathrm{C}(760 \mathrm{mmHg}) ; v_{\max }$ (neat, $\mathrm{cm}^{-1}$ ) 3281, 2942, 2827, 2780, $1659(\mathrm{C}=\mathrm{O}), 1625,1536,1407$, 1230, 1029; ${ }^{1} \mathrm{H}$ NMR (400 MHz, $\mathrm{CDCl}_{3}$ ) $\delta 2.24(\mathrm{~s}, 6 \mathrm{H}), 4.05$ (d, $J 6.4 \mathrm{~Hz}, 2 \mathrm{H}$ ), 5.62 (dd, J 1.6, 10.2 Hz, 1H), 6.12 (dd, J 10.2, 17.0 $\mathrm{Hz}, 1 \mathrm{H}$ ), 6.26 (dd, $J 1.6,17.0 \mathrm{~Hz}, 1 \mathrm{H}), 6.71-6.82$ (brs, $1 \mathrm{H}) ;{ }^{13} \mathrm{C}$ NMR (101 MHz, $\mathrm{CDCl}_{3}$ ) $\delta 41.8(\mathrm{Me}), 61.5,126.0$ (both $\mathrm{CH}_{2}$ ), $130.7(\mathrm{CH}), 166.2(\mathrm{C}=\mathrm{O})$; HRMS (ESI) $m / z[\mathrm{M}+\mathrm{H}]^{+}, \mathrm{C}_{6} \mathrm{H}_{13} \mathrm{~N}_{2} \mathrm{O}$, calcd 129.1028, observed 129.1026.

$\mathrm{N}$-[(Dimethylamino)methyl]-2-methylprop-2-enamide (8b). (32.10 g, 90\%) colourless liquid, bp 60-62 ${ }^{\circ} \mathrm{C}(0.25 \mathrm{mmHg})$; $v_{\max }\left(\right.$ neat, $\left.\mathrm{cm}^{-1}\right)$ 3323, 2942, 2827, $1658(\mathrm{C}=\mathrm{O}), 1619,1523$, 1453, 1311, 1196, 1049, 1033; ${ }^{1} \mathrm{H}$ NMR (400 $\mathrm{MHz}, \mathrm{CDCl}_{3}$ ) $\delta 1.92(\mathrm{~s}, 3 \mathrm{H}), 2.23(\mathrm{~s}, 6 \mathrm{H}), 4.03(\mathrm{~d}, J 6.3 \mathrm{~Hz}, 2 \mathrm{H}), 5.30(\mathrm{~s}, 1 \mathrm{H})$, 5.66 (s, 1H), 6.31-6.45 (brs, $1 \mathrm{H}) ;{ }^{13} \mathrm{C} \mathrm{NMR} \mathrm{(101} \mathrm{MHz,} \mathrm{CDCl}_{3}$ ) $\delta$ 18.7, 42.3 (both Me), 62.2, 119.5 (both $\mathrm{CH}_{2}$ ), 140.1 (C), 169.0 $(\mathrm{C}=\mathrm{O})$; HRMS (ESI) $\mathrm{m} / z[\mathrm{M}+\mathrm{H}]^{+}, \mathrm{C}_{7} \mathrm{H}_{15} \mathrm{~N}_{2} \mathrm{O}$ calcd 143.1184, observed 143.1180.

$\boldsymbol{N}$-[(Diethylamino)methyl]prop-2-enamide (9a). (32.39 g, $83 \%$ ) yellow oil; bp $65-67{ }^{\circ} \mathrm{C}(0.25 \mathrm{mmHg}) ; v_{\max }$ (neat, $\mathrm{cm}^{-1}$ ) 3289, 2969, 2828, 1657 (C=O), 1624, 1536, 1464, 1233, 1206, 1067; ${ }^{1} \mathrm{H}$ NMR (400 MHz, $\left.\mathrm{CDCl}_{3}\right) \delta 1.08(\mathrm{t}, J 7.2 \mathrm{~Hz}, 6 \mathrm{H}), 2.56$ (q, J 7.2 Hz, 4H), 4.29 (d, J 6.1 Hz, 2H), 5.64 (dd, J 1.4, $10.2 \mathrm{~Hz}$, 1H), 5.90-5.99 (brs, 1H), 6.09 (dd, J 10.2, $17.0 \mathrm{~Hz}, 1 \mathrm{H}), 6.28$ (dd, $J$ 1.4, $17.0 \mathrm{~Hz}, 1 \mathrm{H}) ;{ }^{13} \mathrm{C} \mathrm{NMR}\left(101 \mathrm{MHz}, \mathrm{CDCl}_{3}\right) \delta 12.7$ (Me), 45.4, 56.9, 126.6 (all $\left.\mathrm{CH}_{2}\right), 131.0(\mathrm{CH}), 166.1(\mathrm{C}=\mathrm{O})$; HRMS (ESI) $m / z[\mathrm{M}+\mathrm{H}]^{+}, \mathrm{C}_{8} \mathrm{H}_{17} \mathrm{~N}_{2} \mathrm{O}$, calcd 157.1341, observed 157.1337.

$\mathrm{N}$-[(Diethylamino)methyl]-2-methylprop-2-enamide

(9b).

(33.21 g, 78\%) colourless liquid, bp $72-74{ }^{\circ} \mathrm{C}(0.25 \mathrm{mmHg})$; $v_{\max }\left(\right.$ neat, $\left.\mathrm{cm}^{-1}\right)$ 3344, 2970, 2827, $1656(\mathrm{C}=\mathrm{O}), 1617,1522$, 1455, 1375, 1197, 1066, 1046; ${ }^{1} \mathrm{H}$ NMR (400 MHz, $\mathrm{CDCl}_{3}$ ) $\delta 1.03(\mathrm{t}, J 7.2 \mathrm{~Hz}, 6 \mathrm{H}), 1.90(\mathrm{~s}, 3 \mathrm{H}), 2.52(\mathrm{q}, J 7.2 \mathrm{~Hz}, 4 \mathrm{H}), 4.22$ (d, J 6.0 Hz, 2H), 5.27 (s, 1H), 5.63 (s, 1H), 6.11-6.21 (brs, 1H); ${ }^{13} \mathrm{C}$ NMR (101 MHz, $\mathrm{CDCl}_{3}$ ) $\delta$ 12.7, 18.8 (both Me), 45.4, 57.3, $119.5\left(\right.$ all $\left.\mathrm{CH}_{2}\right), 140.2(\mathrm{C}), 168.9(\mathrm{C}=\mathrm{O})$; HRMS (ESI) $\mathrm{m} / \mathrm{z}$ $[\mathrm{M}+\mathrm{H}]^{+}, \mathrm{C}_{9} \mathrm{H}_{19} \mathrm{~N}_{2} \mathrm{O}$ calcd 171.1497, observed 171.1789.

$\boldsymbol{N}$-[(Dipropylamino)methyl]prop-2-enamide (10a). (37.80 g, $82 \%$ ) colourless plates, mp 25-26 ${ }^{\circ} \mathrm{C}$; $v_{\max }\left(\right.$ neat, $\mathrm{cm}^{-1}$ ) 3269, 2959, 2930, 1657 (C=O), 1623, 1550, 1457, 1246, 1185, 1069; ${ }^{1} \mathrm{H}$ NMR $\left(500 \mathrm{MHz}, \mathrm{CDCl}_{3}\right) \delta 0.82(\mathrm{t}, J 7.4 \mathrm{~Hz}, 6 \mathrm{H}), 1.44$ (sext, $J 7.4 \mathrm{~Hz}, 4 \mathrm{H}), 2.39(\mathrm{t}, J 7.4 \mathrm{~Hz}, 4 \mathrm{H}), 4.23(\mathrm{~d}, J 6.0 \mathrm{~Hz}, 2 \mathrm{H}), 5.58$ (dd, J 1.6, 10.2 Hz, 1H), 6.11 (dd, J 10.2, 17.0 Hz, 1H), 6.22 (dd, $J$ 1.6, $17.0 \mathrm{~Hz}, 1 \mathrm{H}), 6.28-6.39$ (brs, $1 \mathrm{H}) ;{ }^{13} \mathrm{C}$ NMR $(125 \mathrm{MHz}$, $\left.\mathrm{CDCl}_{3}\right) \delta 11.9(\mathrm{Me}), 20.9,54.0,58.1,126.5\left(\right.$ all $\left.\mathrm{CH}_{2}\right), 131.1(\mathrm{CH})$, $166.1(\mathrm{C}=\mathrm{O})$; HRMS (ESI) $m / z[\mathrm{M}+\mathrm{H}]^{+}, \mathrm{C}_{10} \mathrm{H}_{21} \mathrm{~N}_{2} \mathrm{O}$ calcd 185.1654, observed 185.1663.

$\mathrm{N}$-[(Dipropylamino)methyl]-2-methylprop-2-enamide (10b). (43.62 g, 88\%) colourless liquid, bp 86-88 ${ }^{\circ} \mathrm{C}(0.25 \mathrm{mmHg})$; $v_{\max }\left(\right.$ neat $\left.\mathrm{cm}^{-1}\right)$ 3316, 2959, 2934, 2873, $1655(\mathrm{C}=\mathrm{O}), 1619$, 1524, 1456, 1374, 1183, 1075, 1052; ${ }^{1} \mathrm{H}$ NMR (400 MHz, $\mathrm{CDCl}_{3}$ ) $\delta 0.85$ (t, $J 7.4 \mathrm{~Hz}, 6 \mathrm{H}), 1.46$ (sext, $J 7.4 \mathrm{~Hz}, 4 \mathrm{H}), 1.93(\mathrm{~s}, 3 \mathrm{H})$, $2.42(\mathrm{t}, J 7.4 \mathrm{~Hz}, 4 \mathrm{H}), 4.22$ (d, J 6.0 Hz, 2H), $5.29(\mathrm{~s}, 1 \mathrm{H}), 5.65$ (s, 1H), 6.02-6.15 (brs, $1 \mathrm{H}) ;{ }^{13} \mathrm{C}$ NMR (101 MHz, $\left.\mathrm{CDCl}_{3}\right) \delta$ 11.9, 18.8 (both Me), 20.9, 54.1, 58.5, 119.4 (all $\mathrm{CH}_{2}$ ), 140.3 (C), $168.9\left(\mathrm{C}=\mathrm{O}\right.$ ); HRMS (ESI) $m / z[\mathrm{M}+\mathrm{H}]^{+}, \mathrm{C}_{11} \mathrm{H}_{23} \mathrm{~N}_{2} \mathrm{O}$ calcd 199.1810, observed 199.1800.

$N$-[(Dibutylamino)methyl]prop-2-enamide (11a). (44.56 g, $84 \%$ ) colourless liquid, bp $124-126{ }^{\circ} \mathrm{C}(0.25 \mathrm{mmHg}) ; v_{\max }$ (neat, $\mathrm{cm}^{-1}$ ) 3281, 3069, 2957, 2863, $1658(\mathrm{C}=\mathrm{O}), 1625,1542$, 1456, 1459, 1366, 1180, 1071; ${ }^{1} \mathrm{H}$ NMR (400 MHz, $\mathrm{CDCl}_{3}$ ) $\delta 0.89(\mathrm{t}, J 7.3 \mathrm{~Hz}, 6 \mathrm{H}), 1.24-1.33(\mathrm{~m}, 4 \mathrm{H}), 1.40-1.47(\mathrm{~m}, 4 \mathrm{H})$, $2.45(\mathrm{t}, J 7.5 \mathrm{~Hz}, 4 \mathrm{H}), 4.26(\mathrm{~d}, J 6.0 \mathrm{~Hz}, 2 \mathrm{H}), 5.63(\mathrm{dd}, J$ 1.5, $10.2 \mathrm{~Hz}, 1 \mathrm{H}$ ), 5.88-6.00 (brs, 1H), 6.10 (dd, J 10.2, $17.0 \mathrm{~Hz}, 1 \mathrm{H})$, 6.27 (dd, $J 1.5,17.0 \mathrm{~Hz}, 1 \mathrm{H}) ;{ }^{13} \mathrm{C} \mathrm{NMR}\left(101 \mathrm{MHz}, \mathrm{CDCl}_{3}\right) \delta 14.1$ (Me), 20.7, 30.0, 51.9, 58.3, 126.7 (all $\mathrm{CH}_{2}$ ), $131.0(\mathrm{CH}), 166.0$ $(\mathrm{C}=\mathrm{O})$; HRMS (ESI) $\mathrm{m} / \mathrm{z}[\mathrm{M}+\mathrm{H}]^{+}, \mathrm{C}_{12} \mathrm{H}_{25} \mathrm{~N}_{2} \mathrm{O}$ calcd 213.1967, observed 213.1952.

$\mathrm{N}$-[(Dibutylamino)methyl]-2-methylprop-2-enamide (11b). (49.21 g, 87\%) colourless liquid, bp $133-135{ }^{\circ} \mathrm{C}(0.25 \mathrm{mmHg})$; $v_{\max }\left(\right.$ neat, $\left.\mathrm{cm}^{-1}\right)$ 3325, 2957, 2931, 2872, $1625(\mathrm{C}=\mathrm{O}), 1525$, 1456, 1374, 1296, 1179, 1083, 1034; ${ }^{1} \mathrm{H}$ NMR (400 MHz, $\mathrm{CDCl}_{3}$ ) $\delta 0.89(\mathrm{t}, J 7.4 \mathrm{~Hz}, 6 \mathrm{H}), 1.25-1.34(\mathrm{~m}, 4 \mathrm{H}), 1.40-1.47(\mathrm{~m}, 4 \mathrm{H})$, $1.95(\mathrm{~s}, 3 \mathrm{H}), 2.46$ (t, J 7.4 Hz, 4H), $4.24(\mathrm{~d}, J 5.9 \mathrm{~Hz}, 2 \mathrm{H}), 5.32(\mathrm{~s}$, $1 \mathrm{H}), 5.66(\mathrm{~s}, 1 \mathrm{H}), 5.98-6.06$ (brs, $1 \mathrm{H}) ;{ }^{13} \mathrm{C} \mathrm{NMR}(101 \mathrm{MHz}$, $\mathrm{CDCl}_{3}$ ) $\delta 14.1,18.8$ (both Me), 20.7, 30.0, 51.9, 58.6, 119.4 (all $\mathrm{CH}_{2}$ ), 140.4 (C), $168.9(\mathrm{C}=\mathrm{O})$; HRMS (ESI) $m / z[\mathrm{M}+\mathrm{H}]^{+}$, $\mathrm{C}_{13} \mathrm{H}_{27} \mathrm{~N}_{2} \mathrm{O}$ calcd 227.2123, observed 227.2129. 


\section{X-ray crystallographic studies}

Single crystal X-ray diffraction data were collected using an Oxford Diffraction Xcalibur system operated using the CrysAlisPro software ${ }^{36}$ and the data collection temperature was controlled at $150 \mathrm{~K}$ using a Cryojet system from Rigaku Oxford Diffraction. The crystals were hygroscopic and were first coated in cold paraffin oil before being transferred to the cold stream on the diffractometer. The crystal structures were solved using ShelxT version $2014 / 5,{ }^{37}$ and refined using ShelxL version $2017 / 1^{38}$ both of which were operated within the Oscail software package. $^{39}$

Crystal refinement data for 1-(hydroxymethyl)azocan-1-ium chloride (5b). Colourless crystals, $\mathrm{C}_{8} \mathrm{H}_{18} \mathrm{ClNO}, M=179.68$, monoclinic, space group $P 22_{1} / c, a=11.3190(18), \quad b=$ 10.9194(16), $c=7.7658(10) \AA, \alpha=90, \beta=90.269(13), \gamma=90^{\circ}, V=$ 959.8(2) $\AA^{3}, Z=4, T=150.0(1) \mathrm{K}, \rho_{\text {calcd }}=1.243 \mathrm{~g} \mathrm{~cm}^{-3}$, refinement of 147 parameters on 2345 independent reflections out of 7528 measured reflections $\left(R_{\text {int }}=0.0709\right)$ led to $R_{1}=0.0857$ $(I>2 \sigma(I)), \mathrm{w} R_{2}=0.2182$ (all data), and $S=1.119$ with the largest difference peak and hole of 0.396 and -0.398 e $\AA^{-3}$.

Crystal refinement data for $N$-[(azocan-1-yl)methyl]prop-2enamide hydrochloride (7a.HCl). Colourless needle crystals, $\mathrm{C}_{11} \mathrm{H}_{21} \mathrm{ClN}_{2} \mathrm{O}, M=232.75$, triclinic, space group $P \overline{1}, a=$ 10.1121(7), $b=10.4420(6), c=12.0205(14) \AA, \alpha=95.695(8), \beta=$ 91.086(8), $\gamma=97.671(5)^{\circ}, V=1251.02(19) \AA^{3}, Z=4, T=150.0(1)$ $\mathrm{K}, \rho_{\text {calcd }}=1.236 \mathrm{~g} \mathrm{~cm}^{-3}$, refinement of 271 parameters on 4487 independent reflections out of 7456 measured reflections $\left(R_{\text {int }}=0.0638\right)$ led to $R_{1}=0.0684(I>2 \sigma(I)), \mathrm{w} R_{2}=0.2128$ (all data), and $S=0.979$ with the largest difference peak and hole of 0.852 and -0.705 e $\AA^{-3}$.

Crystallographic data for compounds $\mathbf{5 b}$ and $\mathbf{7 a} \cdot \mathbf{H C l}$ have been deposited with the Cambridge Crystallographic Data Centre with deposition numbers CCDC 1819145 and 1819144 respectively. $\dagger$

\section{Conflicts of interest}

There are no conflicts to declare.

\section{Acknowledgements}

We thank the Minstry of Education of the Kingdom of Saudi Arabia for supporting the PhD of Abdullah Alzahrani, and the Irish Research Council (IRC) for Goverment of Ireland Postdoctoral Fellowships for Styliana I. Mirallai and Benjamin A. Chalmers.

\section{Notes and references}

1 V. C. Mannich and W. Krösche, Arch. Pharm., 1912, 250, 647-667, DOI: 10.1002/ardp.19122500151.

2 F. F. Blicke, The Mannich Reaction, Org. React., 2011, 1(10), 303-341, DOI: 10.1002/0471264180.or001.10.
3 V. E. Müller, K. Dinges and W. Graulich, Die Makromol. Chem., 1962, 57, 27-51, DOI: 10.1002/macp.1962. 020570103.

4 V. E. Müller and H. Thomas, Angew. Makromol. Chem., 1973, 34, 111-133, DOI: 10.1002/apmc.1973.050340108.

5 R. C. Baltieri, L. H. Innocentini-Mei, W. M. S. C. Tamashiro, L. Peres and E. Bittencourt, Eur. Polym. J., 2002, 38, 57-62, DOI: 10.1016/S0014-3057(01)00177-X.

6 M. L. Eritsyan, Z. B. Barsegyan, R. A. Karamyan, S. M. Manukyan, T. D. Karapetyan and K. A. Martirosyan, Russ. J. Appl. Chem., 2011, 84, 1257-1260, DOI: 10.1134/ S1070427211070238.

7 E. C. Cho, J. Lee and K. Cho, Macromolecules, 2003, 36, 9929-9934, DOI: 10.1021/ma034851d.

8 D. Roy, W. L. A. Brooks and B. S. Sumerlin, Chem. Soc. Rev., 2013, 42, 7214-7243, DOI: 10.1039/c3cs35499g.

9 Z. Song, K. Wang, C. Gao, S. Wang and W. Zhang, Macromolecules, 2015, 49, 162-171, DOI: 10.1021/ acs.macromol.5b02458.

10 K. Wang, Z. Song, C. Liu and W. Zhang, Polym. Chem., 2016, 7, 3423-3433, DOI: 10.1039/c6py00526h.

11 K. Zhou, Y. Wang, X. Huang, K. Luby-Phelps, B. D. Sumer and J. Gao, Angew. Chem., Int. Ed., 2011, 50, 6109-6114, DOI: 10.1002/anie.201100884.

12 H.-J. Li, J.-Z. Du, J. Liu, X.-J. Du, S. Shen, Y.-H. Zhu, X. Wang, X. Ye, S. Nie and J. Wang, ACS Nano, 2016, 10, 6753-6761, DOI: 10.1021/acsnano.6b02326.

13 B. A. Chalmers, C. Magee, D. L. Cheung, P. B. Zetterlund and F. Aldabbagh, Eur. Polym. J., 2017, 97, 129-137, DOI: 10.1016/j.eurpolymj.2017.10.004.

14 B. A. Chalmers, A. Alzahrani, G. Hawkins and F. Aldabbagh, J. Polym. Sci., Part A: Polym. Chem., 2017, 55, 2123-2128, DOI: 10.1002/pola.28607.

15 X. Su, M. F. Cunningham and P. G. Jessop, Polym. Chem., 2014, 5, 940-944, DOI: 10.1039/c3py01382k.

16 H. Heaney, G. Papageorgiou and R. F. Wilkins, Tetrahedron, 1997, 53, 2941-2958, DOI: 10.1016/S0040-4020(96)01174-X.

17 A. Porzelle and C. M. Williams, Synthesis, 2006, 3025-3030, DOI: $10.1055 / \mathrm{s}-2006-942539$.

18 H. Böhme and K. Hartke, Chem. Ber., 1960, 93, 1305-1309, DOI: $10.1002 /$ cber.19600930610.

19 H. Böhme and P. Backhaus, Liebigs Ann. Chem., 1975, 1790-1796, DOI: 10.1002/jlac.197519751007.

20 J. Schreiber, H. Maag, N. Hashimoto and A. Eschenmoser, Angew. Chem., Int. Ed. Engl., 1971, 10, 330-331, DOI: 10.1002/anie.197103301.

21 G. Kinast and L.-F. Tietze, Angew Chem., Int. Ed. Engl., 1976, 15, 239-240, DOI: 10.1002/anie.197602391.

22 N. Abe, F. Fujisaki and K. Sumoto, Chem. Pharm. Bull., 1998, 46, 142-144, DOI: 10.1248/cpb.46.142.

23 N. Pemberton, V. Åberg, H. Almstedt, A. Westermark and F. Almqvist, J. Org. Chem., 2004, 69, 7830-7835, DOI: 10.1021/jo048554y.

24 Y.-Y. Ku, T. Grieme, Y.-M. Pu, A. V. Bhatia and S. A. King, Tetrahedron Lett., 2005, 46, 1471-1474, DOI: 10.1016/ j.tetlet.2005.01.027. 
25 B. R. Buckley, P. C. B. Page, H. Heaney, E. P. Sampler, S. Carley, C. Brocke and M. A. Brimble, Tetrahedron, 2005, 61, 5876-5888, DOI: 10.1016/j.tet.2005.03.130.

26 V. Werner, M. Ellwart, A. J. Wagner and P. Knochel, Org. Lett., 2015, 17, 2026-2029, DOI: 10.1021/acs.orglett. 5 b00801.

27 D. Alker, L. M. Harwood and C. E. Williams, Tetrahedron, 1997, 53, 12671-12678, DOI: 10.1016/S0040-4020(97)007886.

28 H. Möhrle and G. Keller, Z. Naturforsch., B: J. Chem. Sci., 2003, 58, 885-902, DOI: 10.1021/jo01291a032.

29 T. A. Bryson, G. H. Bonitz, C. J. Reichel and R. E. Dardis, J. Org. Chem., 1980, 45, 524-525, DOI: 10.1021/jo0129 $1 \mathrm{a} 032$.

30 C. A. M. A. Huq, S. Fouzia and M. NizamMohideen, Acta Crystallogr., Sect. E: Struct. Rep. Online, 2013, 69, 1766, DOI: 10.1107/S1600536813030559.

31 A. R. Bhat, A. I. Bhat, F. Athar and A. Azam, Helv. Chim. Acta, 2009, 92, 1644-1656, DOI: 10.1002/hlca.200800461.
32 C. Karakus, L. H. Fischer, S. Schmeding, J. Hummel, N. Risch, M. Schäferling and Elisabeth Holder, Dalton Trans., 2012, 41, 9623-9632, DOI: 10.1039/C2DT30835E.

33 K. A. Jensen and L. Henriksen, Acta Chem. Scand., Ser. B, 1975, 29, 877-883, DOI: 10.3891/acta.chem.scand.29b0877.

34 C. Rochin, O. Babot, J. Dunoguès and F. Duboudin, Synthesis, 1986, 228-229, DOI: 10.1055/s-1986-31627.

35 H. Böhme and E. Raude, Chem. Ber., 1981, 114, 3421-3429, DOI: $10.1002 /$ cber.19811141023.

36 CrysAlisPro, 1.171.37.38, Rigaku Corporation, Oxford, UK, 2015. http://journals.iucr.org/e/services/stdswrefs.html.

37 G. M. Sheldrick, Acta Crystallogr., Sect. A: Found. Crystallogr., 2015, 71, 3-8, DOI: 10.1107/S2053273 314026370 .

38 G. Sheldrick, Acta Crystallogr., Sect. C: Cryst. Struct. Commun., 2015, 71, 3-8, DOI: 10.1107/S2053229614024218.

39 P. McArdle, J. Appl. Crystallogr., 2017, 50, 320-326, DOI: $10.1107 / \mathrm{S} 1600576716018446$. 